\title{
Comparative study on two civil engine thrust reversers for their maintainability analysis
}

\author{
Hua-Cong $\mathrm{Xu}^{\mathrm{a}}$, Yang Pan, Yue-Xi Xiong and Jing-Wu He \\ School of Aeronautic Science and Engineering, Beihang University, Beijing, China
}

\begin{abstract}
Trade-offs studies on two kinds of existing engine thrust reverser design were carried out, applying virtual maintenance method and the basic theory of ergonomics. Analysis on the maintainability of the two kinds of configurations, the O-duct thrust reverser owning the integrated propulsion system (IPS) and the traditional portal D-duct thrust reverser, were compared. According to the differences of O-duct and D-duct thrust reverser in the structure, working principle and mode of motion during the maintenance, structure modelling and kinematics simulation of two kinds of thrust reverser were finished in the same space constraints. Taking DELMIA software as the platform, the partial ergonomic research and evaluation including accessibility analysis, visibility analysis, specific disassembling and assembling time estimation and workspace analysis were put forward through the virtual maintenance simulation of two civil engine thrust reversers. Supplied technical reserves on design and selection, scheme analysis and technical evaluations for domestic engine thrust reverser in the future.
\end{abstract}

\section{Introduction}

In addition to undertake tasks like comprising core engine and accessories, engine air supply and exhaust, and aerodynamic shape adjustment to reduce drag, an important function of nacelle is to provide the reverse thrust of aircraft landing, which can be realized by thrust reverser system. Thrust reverser is used in speed reduction during landing and rejected takeoff by changing the direction of airstream in fan duct [1]. Different thrust reverser structures have effects on aerodynamic performance, structural weight and maintainability. Among them, maintainability is the important quality characteristic of aircraft in the stage of product design, which has a decisive effect on the aircraft reliability, efficiency and economy. Beyond that, the pros and cons of maintainability will also directly affect the aircraft ground turnaround time and attendance [2]. Therefore, to analyse its maintainability is of great significance when it comes to engine thrust reverser performance. The quality of maintainability must be evaluated and validated through the maintainability design analysis and tasks simulation. In this paper, taking a certain type of aircraft as an example, two kinds of maintainability models of thrust reverse configurations have been established in the same space constraints and digital simulation methods have been applied. Virtual maintenance method and basic theory of ergonomics are also used to make comparison in maintainability analysis and evaluation taking DELMIA software as a platform.

\footnotetext{
${ }^{a}$ Corresponding author : 1210552073@qq.com
}

(C) The Authors, published by EDP Sciences. This is an open access article distributed under the terms of the Creative Commons Attribution License 4.0 (http://creativecommons.org/licenses/by/4.0/). 


\section{Structure and maintainability analysis of two kinds of thrust reversers}

Maintenance is the abbreviation of maintain and repair on the aviation engine. Aero engine maintainability refers to ease of engine maintenance [3]. This paper focuses on the influence of two different engine thrust reverse structures named D-Duct and O-Duct in engine maintenance, including inspection of core engine and thrust reverser, maintenance accessibility, time estimation and so on.

\subsection{Introduction on traditional D-Duct type thrust reverser}

\subsubsection{Structure analysis on D-Duct}

The traditional D-Duct type thrust reverser system is also called the grid-baffles thrust reverser, which is composed of left and right $\mathrm{T} / \mathrm{R}$ halves attaching to the engine strut, including translating cowl, inner cowl, cascades, blocker doors, hydraulic actuators, torque box, upper and lower sliders and tracks [4]. The two sleeves are connected by tension latches and inner V-blades, working simultaneously but independent of each other. Figure 1 is the structure of D-Duct thrust reverser.

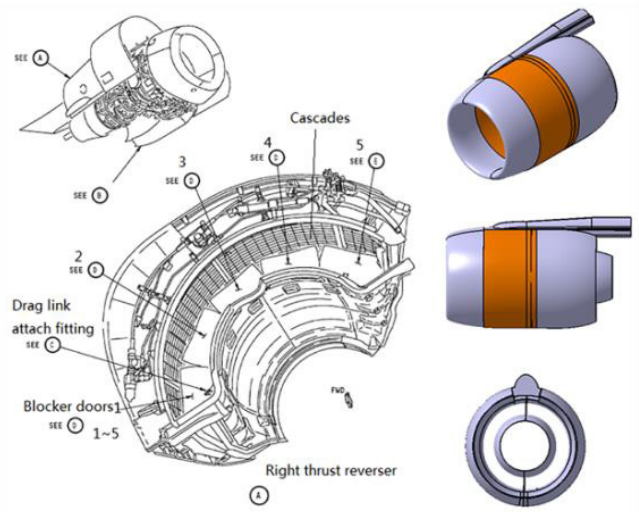

Figure 1. Structure of D-Duct thrust reverser.

\subsubsection{Maintenance methods}

When in maintenance, the thrust reverser is opened on the ground, and the fan cowl and translating cowl are installed on the pylon. The left and right halves are lifted from bottom to upper after releasing the latches under the sleeves, then the fan case, core engine, low-pressure turbines and other components are able to be inspected directly. The core engine including the inlet cowl can be removed or installed while the D-Duct cowl and fan cowl are left on the pylon when in removal/installation tasks. So the traditional D-Duct thrust reverser cowl has satisfied accessibility and visibility, and the operation is simple. Now we will do structure and maintainability analysis for new structure of $\mathrm{O}$ Duct thrust reverser. The following in figure 2 are in maintenance position and latches structure.
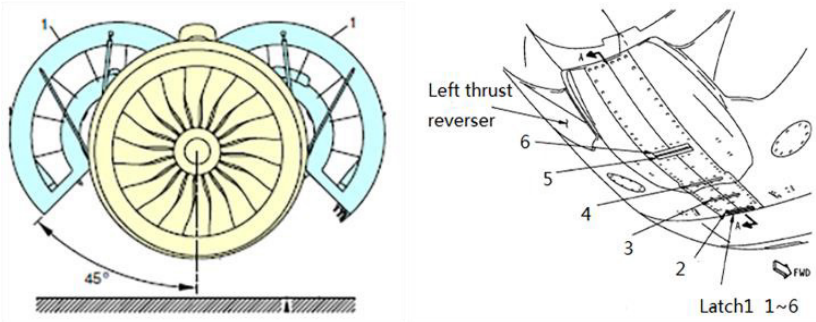

Figure 2. Maintenance position and tension latches of D-Duct. 


\subsection{Introduction on new O-Duct type thrust reverser}

\subsubsection{Structure analysis on O-Duct}

The biggest difference of the two thrust reversers is that the new component has a next-generation integrated propulsion system(IPS) with a low drag front end with a directed flow nozzle deicing system; a fan cowl structurally integrated to the engine; an integrated mounting system for reduced engine distortion and enhanced on-wing performance; and an all-new translating O-Duct thrust reverser configuration. As figure 3 shows, the O-Duct system uses one-piece composite "O" duct, which replaces the two-piece " $\mathrm{D}$ " doors on traditional thrust reversers. When the thrust reverser is working, the entire O-Duct moves afterward to the reverse thrust position driven by electric thrust reverse actuated system(ETRAS), thereby eliminating the need for drag links in the secondary flowpath.
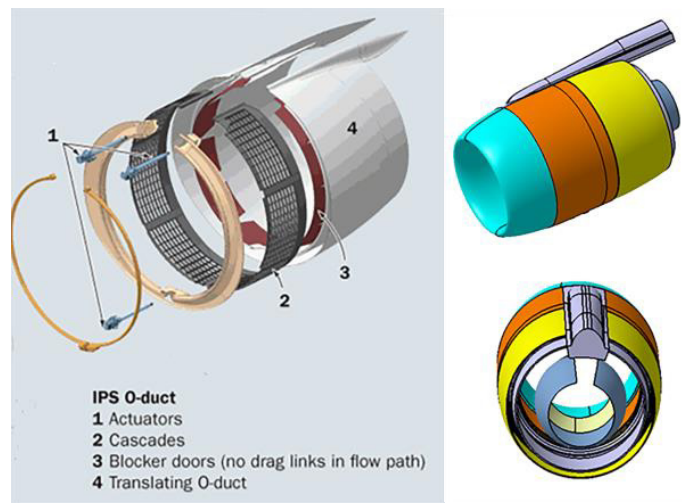

Figure 3. Structure of O-Duct thrust reverser.

\subsubsection{Technique innovation on maintain}

As showed in figure 3, a key feature of the O-Duct is its monolithic structures, 330-deg. carbon composite inner skin. This fact not only reduces the structural weight but also solve a persistent maintenance issue on D-Duct, for the O-Duct has no tension latch, no beam or lower bifurcation on the bottom of translating cowl, neither does lower track liner. Therefore thrust reverser with O-Duct of translating cowl may have easier operation and less maintain in routine maintenance.

Comparing with D-Duct, the integral translating cowl moves only along top slider automatically to intermediate maintenance positions for ease O-Duct maintenance or maintenance positions. What's remarkable about the movement track is that the cowl opening system (COS) and thrust reverse actuated system (TRAS) can move respectively but the moving distance has been limited with slider length and maintenance stop. So we do further research on O-Duct for its maintainability aspects, by studying the simulation of virtual workers for maintenance activities in virtual environment in DELMIA. Then maintainability evaluation can be made to find impact on accessibility analysis, visibility analysis, disassembling and assembling time estimation and so on if we choose O-Duct.

\section{Application of DELMIA-virtual maintenance on thrust reverser}

\subsection{Virtual maintenance introduction and ergonomics application}

With the development of virtual reality technology, applications of virtual maintenance technology in the field of maintainability design, maintenance support capability and maintenance training are on the increase. Traditional maintainability analysis usually requires demonstration of maintenance works, 
which means simulating the maintenance process of real products on physical prototype in actual maintenance environment [5]. Then analysis on visibility, accessibility, mechanics performance for maintenance personnel should be done, while problems occur that work lags behind product design and personal security can not be guaranteed. After virtual maintenance introduced, maintainability design personnel can carry out maintainability analysis and evaluation in parallel; detect problems and defects timely; reduce impact of human factor on aviation maintenance.

Ergonomics is a subject to study the interrelation among human, machine and environment, aiming to reach the optimization results for work efficiency and human-machine security, healthy and comfortable [6]. We combine ergonomics and virtual simulation together to demonstrate maintain works and apply the results to maintainability analysis.

\subsection{Virtual maintenance simulation scene construction}

\subsubsection{The digital prototype model establishment}

Virtual prototype is a maintenance object of virtual maintenance operations, which belongs to the three-dimensional solid model. The disassembly and assembly work of virtual prototype is usually involved in maintenance simulation [7]. We install the two different structures of the nacelles (O-Duct and D-Duct) on a certain type of civil aircraft as the digital prototype, adopting the same installation position, height and outline dimension profile(such as outer contour diameter of inlet cowl, fan cowl and thrust reverser cowl). It can be assured that the result differences are mainly caused by the different structures in the same spatial constraints conditions.

Due to many design attributes included during modelling, miscellaneous data may affect the processing speed. So the model is simplified appropriately to remove geometric information of parts which maintenance task does not involve. At the same time, we eliminate some of the complex parts of the task without affecting the modelling, because the aircraft, especially the engine structure is very complex.

\subsubsection{Maintenance personnel modelling}

Virtual human body model is an important part in maintenance tasks, it is needed to complete all of the ergonomics analysis. Use the Human Builder module in DELMIA to create the full size model of the maintenance personnel, $50^{\text {th }}$ percentile, Taiwan. The establishment of the human body model needs to consider the NASA human element engineering specification. The change of human body posture can be achieved by the manipulation of joint movement, in which complex human body structure includes 68 joints, 6 pairs couple points and 148 degrees of freedom for real joint motion and restriction, in addition, human operator IK(inverse kinematics) function based on forward and reverse kinematics [8].

\subsubsection{Maintenance tools and equipment}

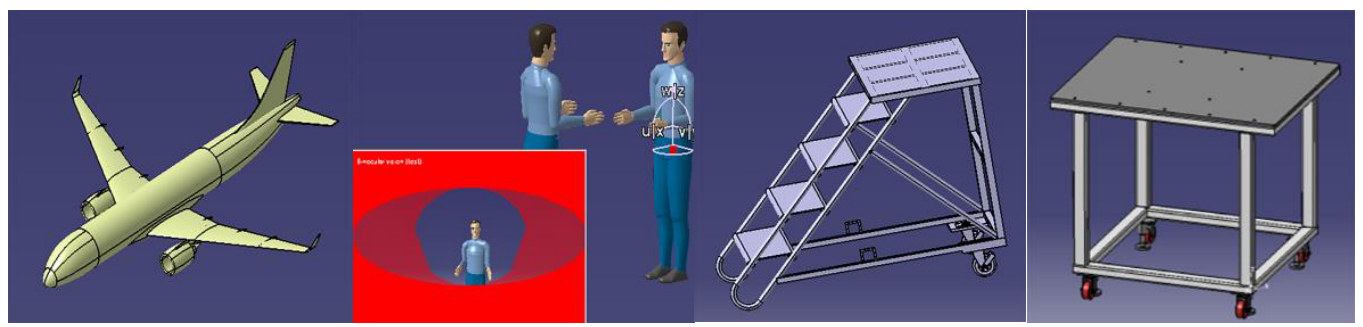

Figure 4. Virtual maintenance environment elements. 
The choice of maintenance tools and equipment shall be subject to the specific maintenance tasks. As is shown in the picture 4, take the operation of removal/installation of translating cowl as an example in daily inspection and maintenance, which divided into two cases of O-Duct/D-Duct, and there are some differences between the required equipment. Through the analysis of model maintenance task, ensure the safeguards equipment and tools for implementation of process.

D-Duct: working ladder operating platform hydraulic pump minitype trunk

O-Duct: working ladder operating platform special ground support equipment(GSE)

\subsection{Process of virtual maintenance simulation}

The virtual simulation process mainly depends on DPM-Assembly process simulation and Human Task Simulation module in DELMIA. The maintenance activities and process simulation are actualized by action of the digital prototype and manikin. It's a dynamic environment as reconstruction of maintenance activities under virtual environment.

\subsubsection{Maintenance example on O-Duct}

Firstly, finish the simulation for inspection on O-Duct. Load the service material into the P.P.R tree, and all process nodes of simulation are established under ProcessList, the digital prototype model is loaded into ProductList, manikins, support equipment, area and others are placed on the ResourceList. Open the fan cowl doors before open the thrust reverser cowl.

Then move the thrust reverser cowl.

(1) Make cautions before opening. Make sure that the thrust reverser can't be operated.

(2) Open the lock at 6 O'clock structure.

(3) Then the translating cowl and cascade components move rearward to maintenance position actuated by two synchronized COS.

Next open the core cowl. The core cowl is composed of two parts: one is FCC (Forward Core Cowl), and the other is ACC (Afterward Core Cowl). FCC is D-type and divided into LH(left half) and $\mathrm{RH}$ (right half), it has three latches at the bottom of FCC. ACC or called rear sliding cowl is Otype like translating cowl, which can move backward along the track.

(1) Open the main latch assurance door and release the bottom latches.

(2) Open FCC. Remove the forward HOR and attached to fan case on one end and IFS on other end.

Then attach afterward HOR, one end detaches from TB and the other attaches to IFS.

(3) Move the ACC backward along the slider.

The O-Duct translating cowl must be removed if need further repair on core engine or thrust reverser.

(1) Front and rear fairings have to be removed before removing O-Duct from pylon.

(2) Release stop bolt at rear of pylon.

(3) The special GSE shall be used to hold out the cowl and carry it away from pylon.

(4) Inspection of core engine. Here are the simulation scene and PETR chart.

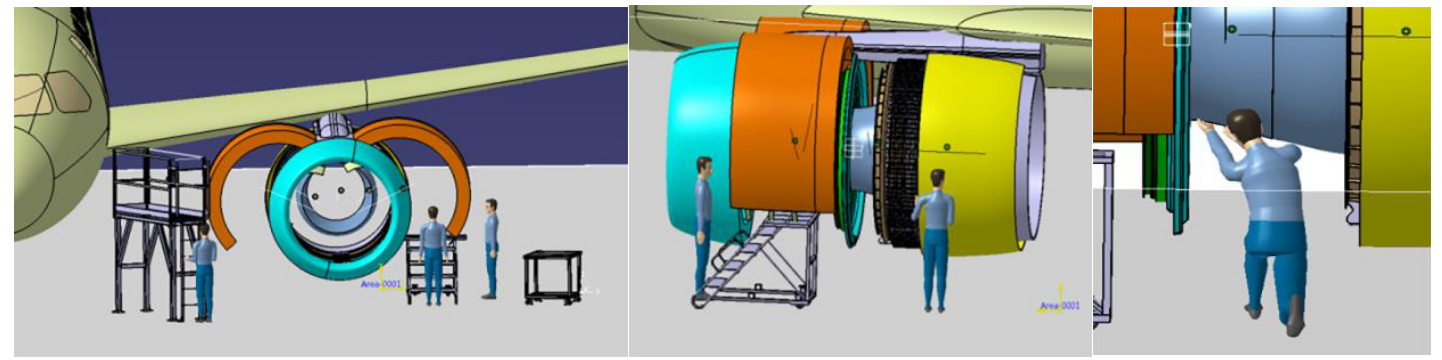

Figure 5. Simulation scene of O-Duct maintenance. 


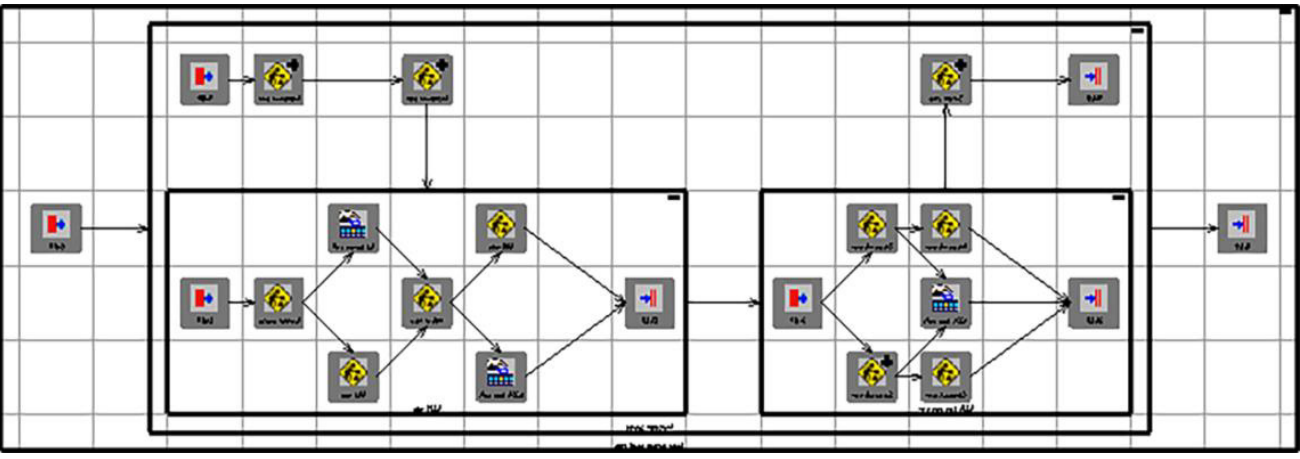

Figure 6. PETR chart of inspection on O-Duct.

The figure 5 shows the simulation scene of O-Duct maintenance, and the figure 6 is PETR chart of inspection on O-Duct.

\subsubsection{Maintenance example on D-Duct}

Compared with O-Duct in above process, D-Duct has the same operation when open the fan cowl doors, for both O-Duct and D-Duct has D-type form. There are some distinctions in opening the thrust reverser cowl, as D-Duct consists of left and right translating sleeves. The procedures to open the cowl are shown in figure 7.

(1) Make cautions before opening.(same with O-Duct)

(2) Unlock the latches along the lower edge of the cowls.

(3) Connect the hand pump.

(4) Operate the hand pump to open the half cowling to the normal maintenance position. The doors can be opened to 33 degrees with the wing leading edge slats extended. Maximum opening is 45 degrees.

(5) Inspection of core engine

Maintenance task:

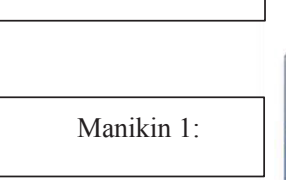

Manikin 2:
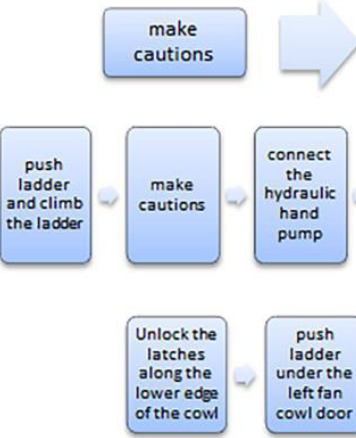
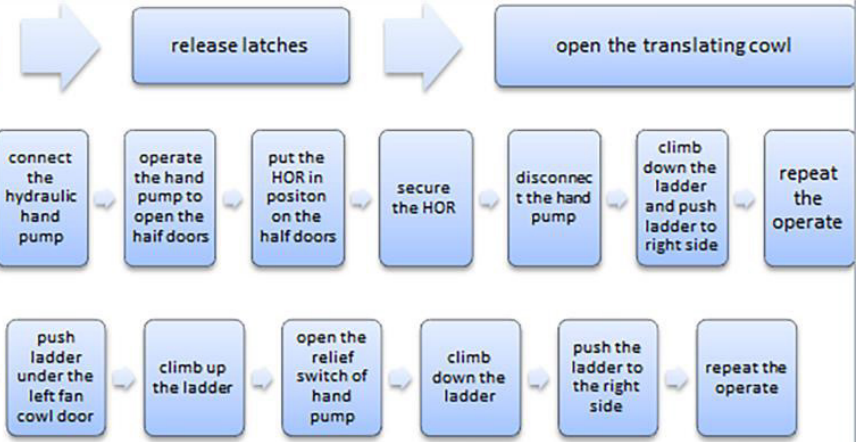

Figure 7. Maintenance program on D-Duct thrust reverser.

\section{Maintainability analysis and evaluation}

\subsection{Accessibility analysis}

Maintenance accessibility is the measurement of difficult degree for reaching target components when repairing product [9]. Including entity reaching (for example a region of the body or by means of tools get in touch with the maintenance position), as well as enough operational space. 


\subsubsection{Qualitative analysis process}

Qualitative analysis for accessibility is conducted by observing the virtual maintenance process, and adjusting manikin's working posture to make maintenance object to reachable range. If the maintenance object is out of range, readjust the position of manikin to find the suitable position and new point of view. Maintenance design may be defective when maintain manikin fails to touch the target or difficult to do that after the adjustment. Figure 8 shows the reachable envelope and accessibility analysis for O-Duct and D-Duct.
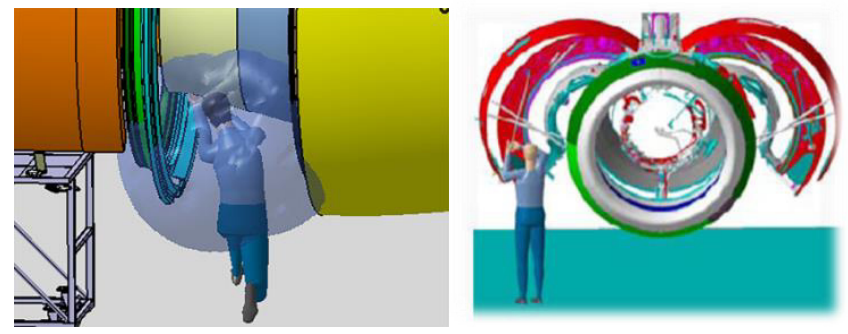

Figure 8. Analysis of reachable envelope and accessibility.

From the analysis of upper envelope diagram, the operated positions are all covered by reachable envelope upper limb, so physical accessibility can be achieved in O-Duct maintenance. However, compared with D-Duct, more operations need to be finished with O-Duct in maintenance tasks. So DDuct performs better than O-Duct in accessibility theoretically for its inner wall connected with translating cowl by the upper and lower bifurcation. When the translating cowl is opened as the picture shows, fan case and core engine can be checked easily. And engine removal/installation is also simple after that. When it comes to O-Duct, removal of translating cowl, FCC and ACC leads to more complex works. Here we need some quantitative analysis

\subsubsection{Quantitative analysis process}

Quantitative analysis for accessibility can be measured by reachable coefficient $K_{\pi}$

$$
K_{\pi}=1-\frac{n_{\pi}}{n_{0}+n_{\pi}}
$$

$n_{0}$ is the basic operation time of maintenance task (refers to most simple action in the process of work). $n_{\pi}$ is the basic operation time of additional task (disassembly of interference part). As can be seen in picture , $n_{0}=17, n_{\pi}=5$, so $K_{\pi}=0.77$, the value is in a specific interval $[0.75,1]$, so we regard maintenance process as in good accessibility. $K_{\pi}=0.81$ in the task of inspection of core engine in DDuct, better than in O-Duct.

\subsection{Collision and interference analysis}

Collision and interference qualitative analysis can be judged directly during virtual maintenance process. Quantitative evaluation can be assessed by $\mathrm{r}$.Measuring and calculating workspace V enclosed with dashed line in virtual environment. Rectangular the minimum workspace $V_{\min }$ related to a certain part of body and tools [10].

$$
r=\frac{V}{V_{\min }}
$$

The maintenance part workspace ratio is good if $r>1.5$, and generally not having collision and interference in the process of maintenance. Obviously, the maintenance part has good workspace both in O-Duct and D-Duct. 


\subsection{Visibility analysis}

DELMIA has vision window function in Human Task Simulation module which can judge whether operational position is entirely visible or in best visual area. As is seen in picture 9, visual range can be intuitively observed on different position through the manikin's eyes. Specific operation should be seen during maintain time, besides enough space to accommodate maintenance manikin's hands and arms, a proper gap is available to observe the target.

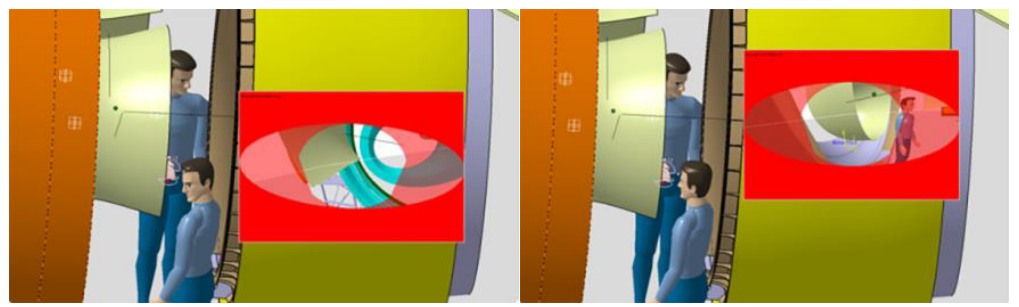

Figure 9. Analysis of visibility.

Comparative study has been made during the simulation to get the conclusion that D-Duct owns better vision as less barrier structure in the task.

\subsection{Disassembling and assembling time estimation}

Disassembling and assembling time can be estimated by three methods: checking simulation process Gantt graph; studying the time when demonstrating the whole simulation process; or examining "simulation periods time" in maintenance process simulation [11]. The Gantt chart in figure 10 is the most intuitive way and can show all the time information of sub-process and entire process, sequence of activities is also obtained through Gantt chart.
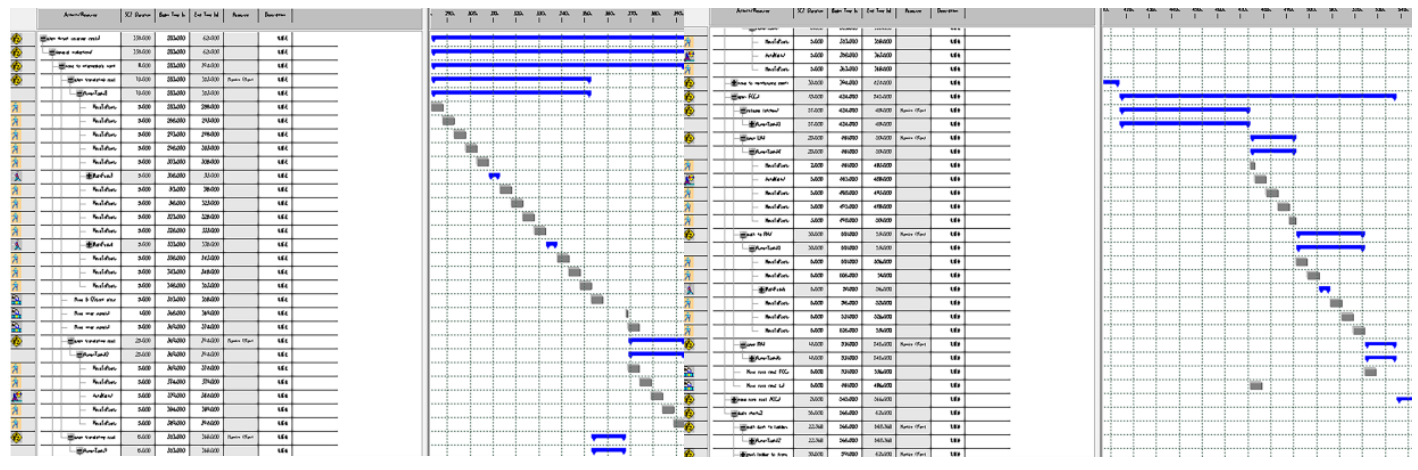

Figure 10. Each motion consume time in Gantt graph.

The maintenance time is not only an important indicator to measure the quality of maintenance, but also an important basis for maintenance hours and maintenance evaluation. Comparing the operation time on two devices, O-Duct costs more time in opening thrust reverser cowl.

\subsection{Working posture analysis}

DELMIA software can generate report and finish maintain efficiency analysis on main maintenance work posture(raise/place analysis, push/pull analysis, walk, carry analysis, biomechanical and so on). Analysis report is to judge if the maintenance person is in suitable working posture, and whether the maintenance operation would decrease the maintenance person's work efficiency or have some behavior leading to fatigue [12]. 


\section{Concluding remarks}

Higher request on engine nacelle design and performance has been put forward since the majority of civil trunk airliners adopt wing-mounted engine structure in modern society. [13] The new type nacelle owing IPS with O-Duct of the translating cowl has different performance compared with traditional D-Duct thrust reverser. Analysis has been done through the maintainability design analysis and tasks simulation in DELMIA. In this paper, information about product's accessibility, rationality of the space distribution and the time to accomplish certain tasks are evaluated by means of virtual maintenance. Since a lot of new technology has been introduced in those O-Duct thrust reversers, they will require highly specialized repairs, and more detailed information has to be obtained by concrete feedback from airline companies.

\section{References}

1. Y.F. Zhang, H.X. Chen, S. Fu, et al., CJA. 33(11), 1993-2001 (2012)

2. H.Z. Wang, Q.G. Zhai, J.F. Yu, et al., Journal of Civil Aviation University of China. 27(4), 56-59 (2009)

3. W. Wang, Engineering Science and Technology II, S1 (2011)

4. J. Ke, X.F. Qi, P. Wang, et al., Civil Aircraft Design \& Research, 108, 14-17 (2013)

5. Z.H. Wang, Q.G. Zhai, Mechanical \& electrical technology, 36-53 (2009)

6. Z.H. Wang, Q.G. Zhai, Journal of Changsha Aeronautical Vocational and Technical College, 9 (4), 36-41 (2009)

7. Z.G. Guo, Z.X. Liu, H. Li, Aircraft Design, 32(3), 62-66 $(2012,6)$

8. W. Jiang, Master Theses of NUDT $(2009,6)$

9. Y.Z. Sun and H.W. Deng, Journal of Traffic and Transportation Engineering, 6(1), 93-112 (2006)

10. Z.X. Yang, Master Theses of JUST

11. H. Liu, J.P. Hao, Journal of System Simulation, 18(2), 378-394 (2006)

12. A. Amundarain, Mecanique \& Industries, 5, 4 (2004)

13. X.F. Zhao, S.G. Tan, W. Shen, et al., Advences in Aeronautica Science and Engineering, 4(3), 268-273 (2013) 